\title{
Parameter estimation of a shifted Wiener sheet
}

\author{
SÁNDOR BARAN ${ }^{a, *}, \quad$ GYULA PAP $^{b}$ and MARTIEN C. A. VAN ZUIJLEN ${ }^{c}$ \\ a Faculty of Informatics, University of Debrecen, H-4010 Debrecen, P.O.Box 12, \\ Hungary; \\ ${ }^{b}$ Bolyai Institute, University of Szeged, H-6720 Szeged, Aradi vértanúk tere 1, \\ Hungary; \\ c Institute for Mathematics, Astrophysics and Particle Physics, \\ Radboud University Nijmegen, Toernooiveld 1, 6525 ED Nijmegen \\ The Netherlands \\ (November 2009)
}

An explicit form is given for the maximum likelihood estimator of a shift parameter $m$ of a shifted Wiener sheet $W(s, t)+m g(s, t),(s, t) \in \mathbb{R}_{+}^{2}$, based on observations $\{W(s, t)+m g(s, t):(s, t) \in G\}$, where $g: \mathbb{R}_{+}^{2} \rightarrow \mathbb{R}$ is a known constraint function and $G$ has a special shape.

Key words: Wiener sheet; $L^{2}$-Riemann integrals; $L^{2}$-processes along a curve; Radon-Nikodym derivative.

2000 Mathematics Subject Classifications: 60G60; 62F12; 62M10.

\section{Introduction}

The Wiener sheet is one of the most important examples of Gaussian random fields. It has various applications in statistical modelling. Wiener sheet appears as limiting process of some random fields defined on the interface of the Ising model [1], it is used to model random polymers [2], to describe the dynamics of Heath-Jarrow-Morton type forward interest rate models [3] or to model random mortality surfaces [4]. Further, Carter [5] considers the problem of estimation of the mean in a nonparametric regression on a two-dimensional regular grid of design points and constructs a Wiener sheet process on the unit square with a drift that is almost the mean function in the nonparametric regression.

In the present paper we consider a shifted Wiener sheet of the form $W(s, t)+m g(s, t)$, where $g$ is a known function, observed on a domain $G$

*Corresponding author. E-mail: baran.sandor@inf.unideb.hu 
of special shape, and we determine the maximum likelihood estimator (MLE) of the unknown parameter $m$. In principle, the Radon-Nikodym derivative of Gaussian measures might be derived from the general Feldman-Hajek theorem (see, e.g. [6]), but in most of the cases explicit calculations can not be carried out. Further, as $\frac{\partial^{2} W(s, t)}{\partial s \partial t}$ is a two dimensional Gaussian white noise, the problem can also be considered as a parameter estimation in a classical Gaussian signal in white noise model (for the results in one dimension see e.g. [7]). For some special domains $G$ (e.g. $G$ is a rectangle with edges parallel to the coordinate lines, $[8]$ ) this approach directly yields the estimator, but it can not be applied for more complicated domains.

For our particular case of a shifted Wiener sheet the MLE of $m$ is given e.g. in [9]. However, the MLE is expressed as a function of a usually unknown random variable satisfying some characterizing equation. In several cases the exact form of this random variable can be derived by a method proposed by Rozanov [10] based on linear stochastic partial differential equations. Arató [11] used Rozanov's method to find the MLE of the shift parameter of a shifted Wiener sheet observed on a special domain. In [12] the authors considered the model of Arató [11], and applying an essentially simpler direct discrete approach they found the MLE of the shift parameter under much weaker conditions. In the present paper the discrete approach is applied for a more general model and more complicated domain of observations. We note that the results derived in Section 3 for discrete samples and the $L^{2}$-integration theory given in Section 4 might be of independent interest.

\section{The model and the estimator}

Let $\left\{W(s, t):(s, t) \in \mathbb{R}_{+}\right\}$be a standard Wiener sheet. Consider the process $Z(s, t):=W(s, t)+m g(s, t), \quad(s, t) \in \mathbb{R}_{+}$, with some known function $g:$ $\mathbb{R}_{+}^{2} \rightarrow \mathbb{R}$ and with an unknown parameter $m \in \mathbb{R}$. Let $[a, c] \subset(0, \infty)$ and $b_{1}, b_{2} \in(a, c)$. Let $\gamma_{1,2}:\left[a, b_{1}\right] \rightarrow \mathbb{R}$ and $\gamma_{0}:\left[b_{2}, c\right] \rightarrow \mathbb{R}$ be continuous, strictly decreasing functions and let $\gamma_{1}:\left[b_{1}, c\right] \rightarrow \mathbb{R}$ and $\gamma_{2}:\left[a, b_{2}\right] \rightarrow$ $\mathbb{R}$ be continuous, strictly increasing functions with $\gamma_{1,2}\left(b_{1}\right)=\gamma_{1}\left(b_{1}\right)>0$, $\gamma_{2}\left(b_{2}\right)=\gamma_{0}\left(b_{2}\right), \quad \gamma_{1,2}(a)=\gamma_{2}(a)$ and $\gamma_{1}(c)=\gamma_{0}(c)$. Consider the curve $\Gamma:=\Gamma_{1,2} \cup \Gamma_{1} \cup \Gamma_{2} \cup \Gamma_{0}$, where

$$
\begin{array}{rlrl}
\Gamma_{1,2} & :=\left\{\left(s, \gamma_{1,2}(s)\right): s \in\left[a, b_{1}\right]\right\}, & \Gamma_{1}:=\left\{\left(s, \gamma_{1}(s)\right): s \in\left[b_{1}, c\right]\right\}, \\
\Gamma_{2}:=\left\{\left(s, \gamma_{2}(s)\right): s \in\left[a, b_{2}\right]\right\}, & \Gamma_{0}:=\left\{\left(s, \gamma_{0}(s)\right): s \in\left[b_{2}, c\right]\right\},
\end{array}
$$




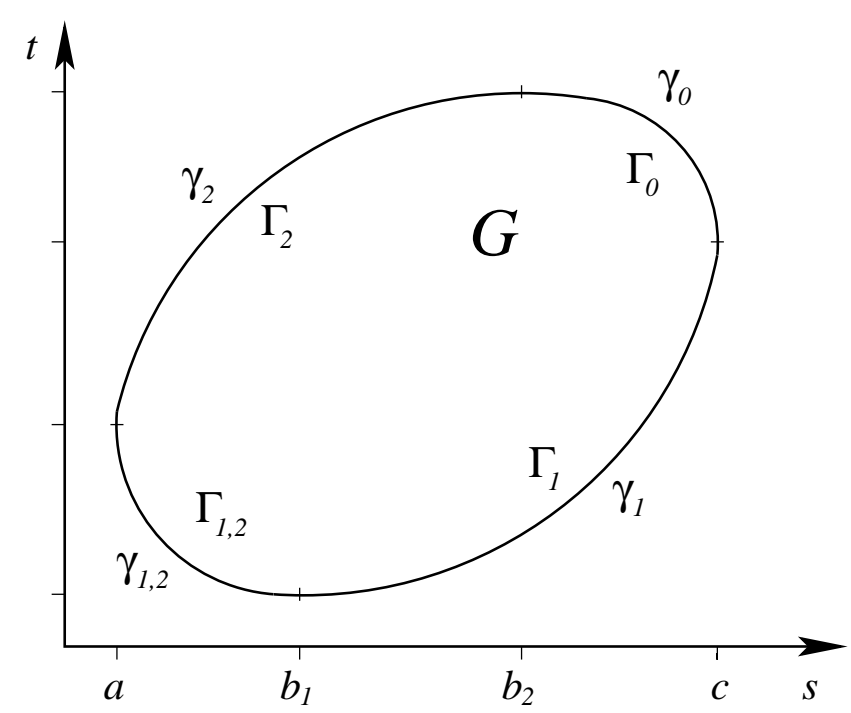

Figure 1. A example of a set of observations $G$.

and for a given $\varepsilon>0$ let $\Gamma_{1,2}^{\varepsilon}, \Gamma_{1}^{\varepsilon}, \Gamma_{2}^{\varepsilon}$ and $\Gamma_{0}^{\varepsilon}$ denote the inner $\varepsilon$-strip of $\Gamma_{1,2}, \Gamma_{1}, \Gamma_{2}$ and $\Gamma_{0}$, respectively, that is e.g.

$$
\begin{aligned}
\Gamma_{1,2}^{\varepsilon}:=\left\{(s, t) \in \mathbb{R}^{2}: s\right. & \in[a, a+\varepsilon], t \in\left[\gamma_{1,2}(s), \gamma_{1,2}(a)\right] \text { or } \\
s & \left.\in\left[a+\varepsilon, b_{1}\right], t \in\left[\gamma_{1,2}(s), \gamma_{1,2}(s)+\varepsilon\right]\right\} .
\end{aligned}
$$

Suppose that there exists an $\varepsilon>0$ such that

$$
\Gamma_{1}^{\varepsilon} \cap \Gamma_{2}^{\varepsilon}=\emptyset \quad \text { and } \quad \Gamma_{1,2}^{\varepsilon} \cap \Gamma_{0}^{\varepsilon}=\emptyset
$$

and consider the set $G:=G_{1} \cup G_{2} \cup G_{3}$, where

$$
\begin{aligned}
& G_{1}:=\left\{(s, t) \in \mathbb{R}^{2}: s \in\left[a, b_{1} \wedge b_{2}\right], t \in\left[\gamma_{1,2}(s), \gamma_{2}(s)\right]\right\}, \\
& G_{2}:=\left\{\begin{array} { l l } 
{ \{ ( s , t ) \in \mathbb { R } ^ { 2 } : s \in [ b _ { 1 } , b _ { 2 } ] , t \in [ \gamma _ { 1 } ( s ) , \gamma _ { 2 } ( s ) ] \} , } & { \text { if } b _ { 1 } \leq b _ { 2 } , }
\end{array} \left\{\begin{array}{ll}
\left\{(s, t) \in \mathbb{R}^{2}: s \in\left[b_{2}, b_{1}\right], t \in\left[\gamma_{1,2}(s), \gamma_{0}(s)\right]\right\}, & \text { if } b_{1}>b_{2},
\end{array}\right.\right. \\
& G_{3}:=\left\{(s, t) \in \mathbb{R}^{2}: s \in\left[b_{1} \vee b_{2}, c\right], t \in\left[\gamma_{1}(s), \gamma_{0}(s)\right]\right\}
\end{aligned}
$$

An example of such a set of observations can be seen of Figure 2.

Theorem 2.1 If $g$ is twice continuously differentiable inside $G$ and the partial derivatives $\partial_{1} g, \partial_{2} g$ and $\partial_{1} \partial_{2} g$ can be continuously extended to $G$ then the probability measures $\mathrm{P}_{Z}$ and $\mathrm{P}_{W}$, generated on $C(G)$ by the sheets 
$Z$ and $W$, respectively, are equivalent and the Radon-Nikodym derivative of $\mathrm{P}_{Z}$ with respect to $\mathrm{P}_{W}$ equals

$$
\frac{\mathrm{dP}_{Z}}{\mathrm{dP}_{W}}(Z)=\exp \left\{-\frac{1}{2}\left(A m^{2}-2 \zeta m\right)\right\},
$$

where

$$
\begin{aligned}
A:= & \frac{g\left(b_{1}, \gamma_{1,2}\left(b_{1}\right)\right)^{2}}{b_{1} \gamma_{1,2}\left(b_{1}\right)}+\int_{a}^{b_{1}} \frac{\left[g\left(s, \gamma_{1,2}(s)\right)-s \partial_{1} g\left(s, \gamma_{1,2}(s)\right)\right]^{2}}{s^{2} \gamma_{1,2}(s)} \mathrm{d} s \\
& +\int_{b_{1}}^{c} \frac{\left[\partial_{1} g\left(s, \gamma_{1}(s)\right)\right]^{2}}{\gamma_{1}(s)} \mathrm{d} s+\int_{\gamma_{1,2}\left(b_{1}\right)}^{\gamma_{1,2}(a)} \frac{\left[\partial_{2} g\left(\gamma_{1,2}^{-1}(t), t\right)\right]^{2}}{\gamma_{1,2}^{-1}(t)} \mathrm{d} t \\
& +\int_{\gamma_{2}(a)}^{\gamma_{2}\left(b_{2}\right)} \frac{\left[\partial_{2} g\left(\gamma_{2}^{-1}(t), t\right)\right]^{2}}{\gamma_{2}^{-1}(t)} \mathrm{d} t+\iint_{G}\left[\partial_{1} \partial_{2} g(s, t)\right]^{2} \mathrm{~d} s \mathrm{~d} t,
\end{aligned}
$$

and

$$
\begin{aligned}
\zeta:= & \frac{g\left(b_{1}, \gamma_{1,2}\left(b_{1}\right)\right) Z\left(b_{1}, \gamma_{1,2}\left(b_{1}\right)\right)}{b_{1} \gamma_{1,2}\left(b_{1}\right)}+\int_{b_{1}}^{c} \frac{\partial_{1} g\left(s, \gamma_{1}(s)\right)}{\gamma_{1}(s)} Z\left(\mathrm{~d} s, \gamma_{1}(s)\right) \\
& +\int_{a}^{b_{1}} \frac{\left[g\left(s, \gamma_{1,2}(s)\right)-s \partial_{1} g\left(s, \gamma_{1,2}(s)\right)\right]}{s^{2} \gamma_{1,2}(s)}\left[Z\left(s, \gamma_{1,2}(s)\right) \mathrm{d} s-s Z\left(\mathrm{~d} s, \gamma_{1,2}(s)\right)\right] \\
& +\int_{\gamma_{1,2}\left(b_{1}\right)}^{\gamma_{1,2}(a)} \frac{\partial_{2} g\left(\gamma_{1,2}^{-1}(t), t\right)}{\gamma_{1,2}^{-1}(t)} Z\left(\gamma_{1,2}^{-1}(t), \mathrm{d} t\right)+\int_{\gamma_{2}(a)}^{\gamma_{2}\left(b_{2}\right)} \frac{\partial_{2} g\left(\gamma_{2}^{-1}(t), t\right)}{\gamma_{2}^{-1}(t)} Z\left(\gamma_{2}^{-1}(t), \mathrm{d} t\right) \\
& +\iint_{G} \partial_{1} \partial_{2} g(s, t) Z(\mathrm{~d} s, \mathrm{~d} t) .
\end{aligned}
$$

The maximum likelihood estimator of the shift parameter $m$ based on the observations $\{Z(s, t):(s, t) \in G\}$ has the form $\widetilde{m}=\zeta / A$ and has a normal distribution with mean $m$ and variance $1 / A$.

The integrals in the expression for $\zeta$ above are meant as $L^{2}$-integrals and will be discussed in Section 4 . 
Remark 1 The form of $A$ and $\zeta$ in Theorem 2.1 is very similar to the form of the corresponding quantities in [13, Theorem 4], where $G=\left[c_{1}, c_{2}\right] \times\left[d_{1}, d_{2}\right]$, with $\left[c_{1}, c_{2}\right],\left[d_{1}, d_{2}\right] \subset(0, \infty)$. This particular result can also be derived from the results of [8]. Moreover, if $g \equiv 1$ one can show that $A$ and $\zeta$ gives back the corresponding quantities of [11, Theorem 2] and [12, Theorem 1.1].

\section{Radon-Nikodym derivatives of finite samples}

Let $0<s_{1}<s_{2}<\cdots<s_{L}$ and $0<t_{1}<t_{2}<\cdots<t_{M}$ be real numbers and let $1=\lambda_{1}<\cdots<\lambda_{n}<\lambda_{n+1}<\cdots<\lambda_{n+m}=L, \quad 1=\lambda_{1}^{\prime}<\cdots<\lambda_{n^{\prime}}^{\prime}<$ $\lambda_{n^{\prime}+1}^{\prime}<\cdots<\lambda_{n^{\prime}+m^{\prime}}^{\prime}=L, \quad 1=\mu_{1}<\cdots<\mu_{n}<\mu_{n+1}<\cdots<\mu_{n+n^{\prime}}=M$ and $1=\mu_{1}^{\prime}<\cdots<\mu_{m}^{\prime}<\mu_{m+1}^{\prime}<\cdots<\mu_{m+m^{\prime}}^{\prime}=M$ be integers,

$$
\begin{aligned}
R & :=\left\{(i, j) \in \mathbb{N}^{2}: 1 \leq i \leq L, 1 \leq j \leq M\right\}, \\
R_{1,2}:= & \bigcup_{k=1}^{n-1}\left\{(i, j) \in \mathbb{N}^{2}: \lambda_{k} \leq i<\lambda_{k+1}, 1 \leq j<\mu_{n-k+1}\right\}, \\
R_{1}:= & \bigcup_{k=n+1}^{n+m-1}\left\{(i, j) \in \mathbb{N}^{2}: \lambda_{k}<i \leq \lambda_{k+1}, 1<j \leq \mu_{k-n+1}^{\prime}\right\}, \\
R_{2}:= & \bigcup_{k=1}^{n^{\prime}-1}\left\{(i, j) \in \mathbb{N}^{2}: \lambda_{k}^{\prime} \leq i<\lambda_{k+1}^{\prime}, \mu_{n+k}<j \leq M\right\}, \\
R_{0}:= & \bigcup_{k=n^{\prime}+1}^{n^{\prime}+m^{\prime}-1}\left\{(i, j) \in \mathbb{N}^{2}: \lambda_{k}^{\prime}<i \leq \lambda_{k+1}^{\prime}, \mu_{n^{\prime}+m^{\prime}+m-k}^{\prime}<j \leq M\right\}, \\
H:= & R \backslash\left(R_{1,2} \cup R_{1} \cup R_{2} \cup R_{0}\right), \\
H_{+}:= & \left\{\left(\lambda_{k}, \mu_{n-k+1}\right): k=1, \ldots, n\right\}, \\
H_{-}:= & \left\{\left(\lambda_{k}, \mu_{n-k+2}\right): k=2, \ldots, n\right\}, \\
H_{1}:= & \left\{\left(i, \mu_{k}\right): k=1, \ldots, n, \lambda_{n-k+1}<i \leq \lambda_{n-k+2}\right\} \\
& \cup\left\{\left(i, \mu_{k}^{\prime}\right): k=2, \ldots, m, \lambda_{n+k-1}<i \leq \lambda_{n+k}\right\}, \\
H_{2}:= & \left\{\left(\lambda_{k}, j\right): k=1, \ldots, n, \mu_{n-k+1}<j \leq \mu_{n-k+2}\right\} \\
& \cup\left\{\left(\lambda_{k}^{\prime}, j\right): k=2, \ldots, n^{\prime}, \mu_{n+k-1}<j \leq \mu_{n+k}\right\}, \\
H_{1,2}:= & H \backslash\left(H_{+} \cup H_{-} \cup H_{1} \cup H_{2}\right) .
\end{aligned}
$$




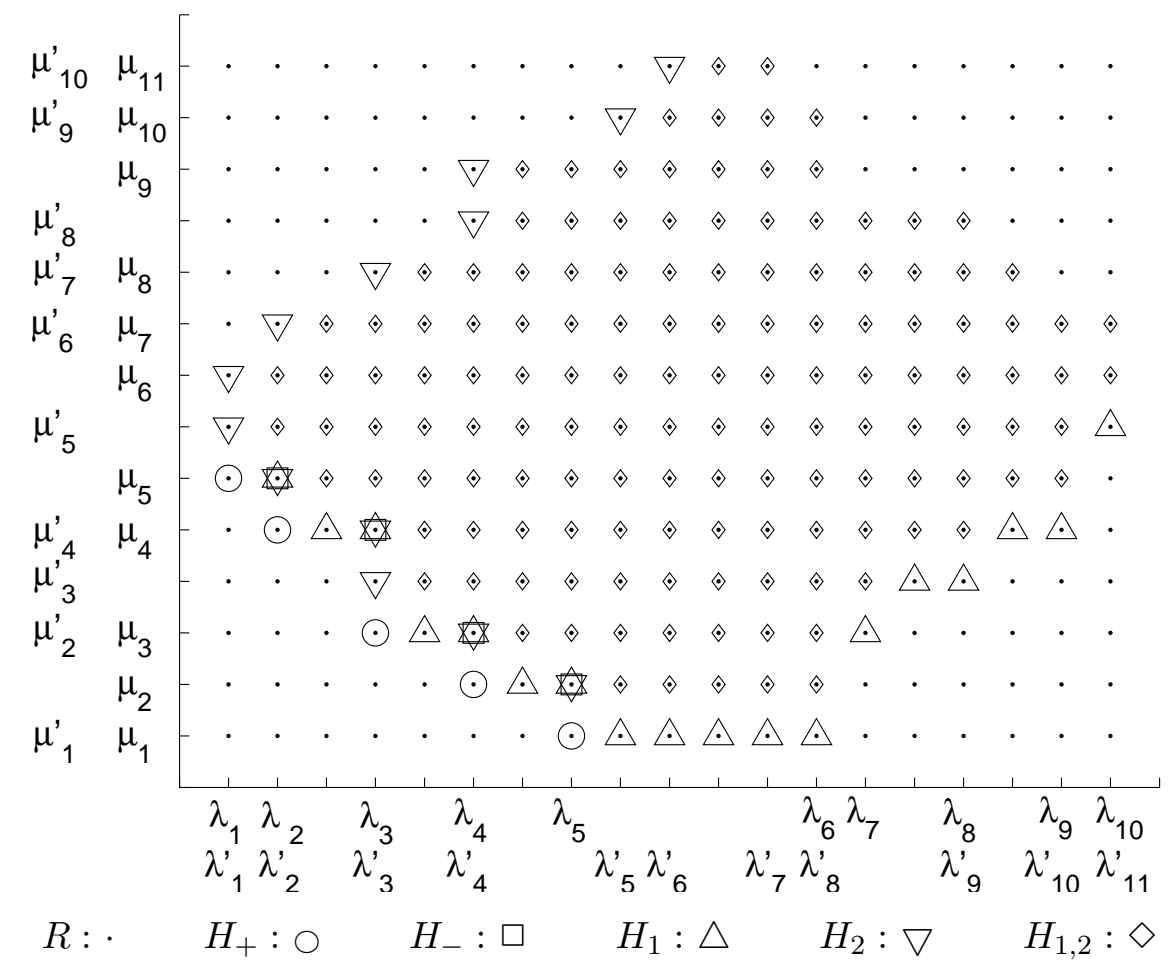

Figure 2. An example of index sets $R, H_{+} \underset{n^{\prime}=6 .}{H_{-}, H_{1}, H_{2}}$ and $H_{1,2}$ for $n=m=m^{\prime}=5$ and

(See Figure 2 for an example.)

Lemma 3.1 The joint density of $\left\{W\left(s_{i}, t_{j}\right):(i, j) \in H\right\}$ is

$$
c \cdot f\left(x_{i, j}:(i, j) \in H_{+} \cup H_{-}\right) \cdot g\left(x_{i, j}:(i, j) \in H\right),
$$

where $c$ is a norming constant,

$$
\begin{aligned}
f\left(x_{i, j}:(i, j) \in H_{+} \cup H_{-}\right)= & \exp \left\{-\sum_{(i, j) \in H_{+}} \frac{x_{i, j}^{2}}{2 s_{i} t_{j}}+\sum_{(i, j) \in H_{-}} \frac{x_{i, j}^{2}}{2 s_{i} t_{j}}\right\}, \\
g\left(x_{i, j}:(i, j) \in H\right)=\exp \{- & \sum_{(i, j) \in H_{1}} \frac{\left(\Delta_{1} x_{i, j}\right)^{2}}{2\left(\Delta s_{i}\right) t_{j}}-\sum_{(i, j) \in H_{2}} \frac{\left(\Delta_{2} x_{i, j}\right)^{2}}{2 s_{i}\left(\Delta t_{j}\right)} \\
& \left.-\sum_{(i, j) \in H_{1,2}} \frac{\left(\Delta_{1} \Delta_{2} x_{i, j}\right)^{2}}{2\left(\Delta s_{i}\right)\left(\Delta t_{j}\right)}\right\}
\end{aligned}
$$


where $\Delta_{1} x_{i, j}:=x_{i, j}-x_{i-1, j}, \quad \Delta_{2} x_{i, j}:=x_{i, j}-x_{i, j-1} \quad$ and $\quad \Delta s_{i}:=s_{i}-s_{i-1}$, $\Delta t_{j}:=t_{j}-t_{j-1}$.

Proof The joint density of $\left\{W\left(s_{i}, t_{j}\right):(i, j) \in R\right\}$ equals

$$
c \exp \left\{-\frac{x_{1,1}^{2}}{2 s_{1} t_{1}}-\sum_{i=2}^{L} \frac{\left(\Delta_{1} x_{i, 1}\right)^{2}}{2\left(\Delta s_{i}\right) t_{1}}-\sum_{j=2}^{M} \frac{\left(\Delta_{2} x_{1, j}\right)^{2}}{2 s_{1}\left(\Delta t_{j}\right)}-\sum_{i=2}^{L} \sum_{j=2}^{M} \frac{\left(\Delta_{1} \Delta_{2} x_{i, j}\right)^{2}}{2\left(\Delta s_{i}\right)\left(\Delta t_{j}\right)}\right\}
$$

which is, in fact, a special case of the statement for $n=m=n^{\prime}=m^{\prime}=1$, $1=\lambda_{1}=\lambda_{1}^{\prime}<\lambda_{2}=\lambda_{2}^{\prime}=L, 1=\mu_{1}=\mu_{1}^{\prime}<\mu_{2}=\mu_{2}^{\prime}=M$, hence for $H=R$, $R_{1,2}=R_{1}=R_{2}=R_{0}=\emptyset$ with $H_{+}=\{(1,1)\}, H_{-}=\emptyset, \quad H_{1}=\{(i, 1): 2 \leq$ $i \leq L\}, \quad H_{2}=\{(1, j): 2 \leq j \leq M\}$, and $H_{1,2}=\{(i, j): 2 \leq i \leq L, 2 \leq j \leq$ $M\}$. From this we calculate the joint density of $\left\{W\left(s_{i}, t_{j}\right):(i, j) \in H\right\}$ by integrating with respect to the variables $\left\{x_{i, j}:(i, j) \in R_{1,2} \cup R_{1} \cup R_{2} \cup R_{0}\right\}$ such that in each step we obtain a set which can be represented in the same form as $H$. Hence, it is sufficient to show that by integrating formula (3.1) for $H$ with respect to a variable $x_{i, j}$ with

(i) $(i+1, j),(i, j+1) \in H$ and $(i-1, j),(i, j-1) \notin H$ (south-west corner), (ii) or $(i-1, j),(i, j+1) \in H$ and $(i+1, j),(i, j-1) \notin H$ (south-east corner), (iii) or $(i, j-1),(i+1, j) \in H$ and $(i-1, j),(i, j+1) \notin H$ (north-west corner), (iv) or $(i, j-1),(i-1, j) \in H$ and $(i+1, j),(i, j+1) \notin H$ (north-east corner), we obtain formula $(3.1)$ for $H \backslash\{(i, j)\}$.

Let us start from the south-west corner, and consider a variable $x_{i, j}$ with $(i, j) \in H_{+}, \quad(i+1, j) \in H$ and $(i, j+1) \in H$. We have only four terms in the exponent of formula (3.1) containing $x_{i, j}$, namely

$$
S_{1}:=-\frac{x_{i, j}^{2}}{2 s_{i} t_{j}}-\frac{\left(\Delta_{1} x_{i+1, j}\right)^{2}}{2\left(\Delta s_{i+1}\right) t_{j}}-\frac{\left(\Delta_{2} x_{i, j+1}\right)^{2}}{2 s_{i}\left(\Delta t_{j+1}\right)}-\frac{\left(\Delta_{1} \Delta_{2} x_{i+1, j+1}\right)^{2}}{2\left(\Delta s_{i+1}\right)\left(\Delta t_{j+1}\right)} .
$$

After forming a full square in the variable $x_{i, j}$ and integrating with respect to $x_{i, j}, S_{1}$ can be replaced by (see [12])

$$
-\frac{x_{i+1, j}^{2}}{2 s_{i+1} t_{j}}-\frac{x_{i, j+1}^{2}}{2 s_{i} t_{j+1}}+\frac{x_{i+1, j+1}^{2}}{2 s_{i+1} t_{j+1}}-\frac{\left(\Delta_{1} x_{i+1, j+1}\right)^{2}}{2\left(\Delta s_{i+1}\right) t_{j+1}}-\frac{\left(\Delta_{2} x_{i+1, j+1}\right)^{2}}{2 s_{i+1}\left(\Delta t_{j+1}\right)} .
$$

This means that formula (3.1) is valid for $H \backslash\{(i, j)\}$.

After integrating with respect to $\left\{x_{i, j}:(i, j) \in R_{1,2}\right\}$, one can continue with the south-east corner. Consider a variable $x_{i, j}$ with $(i, j) \in H,(i-1, j) \in H$ $(i, j+1) \in H$ and $(i, j-1) \notin H, \quad(i+1, j) \notin H$. The part of the exponent 
of (3.1) containing $x_{i, j}$ is

$$
S_{2}:=-\frac{\left(\Delta_{1} x_{i, j}\right)^{2}}{2\left(\Delta s_{i}\right) t_{j}}-\frac{\left(\Delta_{1} \Delta_{2} x_{i, j+1}\right)^{2}}{2\left(\Delta s_{i}\right)\left(\Delta t_{j+1}\right)} .
$$

After integrating with respect to $x_{i, j}$, one can replace $S_{2}$ by

$$
-\frac{\left(\Delta_{1} x_{i, j+1}\right)^{2}}{2\left(\Delta s_{i}\right) t_{j+1}}
$$

so (3.1) is valid for $H \backslash\{(i, j)\}$.

The north-west corner can be handled in a similar way. Consider a variable $x_{i, j} \quad$ with $(i, j) \in H, \quad(i+1, j) \in H, \quad(i, j-1) \in H$ and $(i, j+1) \notin H$, $(i-1, j) \notin H$. The part of the exponent of (3.1) containing $x_{i, j}$ is

$$
S_{3}:=-\frac{\left(\Delta_{2} x_{i, j}\right)^{2}}{2 s_{i}\left(\Delta t_{j}\right)}-\frac{\left(\Delta_{1} \Delta_{2} x_{i+1, j}\right)^{2}}{2\left(\Delta s_{i+1}\right)\left(\Delta t_{j}\right)}
$$

After integrating with respect to $x_{i, j}$, one can replace $S_{3}$ by

$$
-\frac{\left(\Delta_{2} x_{i+1, j}\right)^{2}}{2 s_{i+1}\left(\Delta t_{j}\right)}
$$

so (3.1) is valid for $H \backslash\{(i, j)\}$.

Finally, let us deal with the north-east corner and consider a variable $x_{i, j}$ with $(i, j) \in H,(i-1, j) \in H,(i, j-1) \in H$ and $(i, j+1) \notin H,(i+1, j) \notin H$. It is easy to see, that $x_{i, j}$ is contained only in one term of (3.1), namely in

$$
-\frac{\left(\Delta_{1} \Delta_{2} x_{i, j}\right)^{2}}{2\left(\Delta s_{i}\right)\left(\Delta t_{j}\right)}
$$

which disappears after integration with respect to $x_{i, j}$. Hence, we obtain formula (3.1) for $H \backslash\{(i, j)\}$, completing the induction step.

LemMA 3.2 The probability measures $\mathrm{P}_{Z}^{H}$ and $\mathrm{P}_{W}^{H}$, generated by the random variables $\left\{Z\left(s_{i}, t_{j}\right):(i, j) \in H\right\}$ and $\left\{W\left(s_{i}, t_{j}\right):(i, j) \in H\right\}$, respectively, are equivalent and

$$
\frac{\mathrm{dP}_{Z}^{H}}{\mathrm{dP}_{W}^{H}}\left(x_{i, j}:(i, j) \in H\right)=\exp \left\{-\frac{1}{2}\left(A_{H} m^{2}-2 y_{H} m\right)\right\},
$$


where

$$
\begin{aligned}
A_{H}:= & \sum_{(i, j) \in H_{+}} \frac{g\left(s_{i}, t_{j}\right)^{2}}{s_{i} t_{j}}-\sum_{(i, j) \in H_{-}} \frac{g\left(s_{i}, t_{j}\right)^{2}}{s_{i} t_{j}}+\sum_{(i, j) \in H_{1}} \frac{\left(\Delta_{1} g\left(s_{i}, t_{j}\right)\right)^{2}}{\left(\Delta s_{i}\right) t_{j}} \\
& +\sum_{(i, j) \in H_{2}} \frac{\left(\Delta_{2} g\left(s_{i}, t_{j}\right)\right)^{2}}{s_{i}\left(\Delta t_{j}\right)}+\sum_{(i, j) \in H_{1,2}} \frac{\left(\Delta_{1} \Delta_{2} g\left(s_{i}, t_{j}\right)\right)^{2}}{\left(\Delta s_{i}\right)\left(\Delta t_{j}\right)}, \\
y_{H}:= & \sum_{(i, j) \in H_{+}} \frac{x_{i, j} g\left(s_{i}, t_{j}\right)}{s_{i} t_{j}}-\sum_{(i, j) \in H_{-}} \frac{x_{i, j} g\left(s_{i}, t_{j}\right)}{s_{i} t_{j}}+\sum_{(i, j) \in H_{1}} \frac{\left(\Delta_{1} x_{i, j}\right)\left(\Delta_{1} g\left(s_{i}, t_{j}\right)\right)}{\left(\Delta_{i}\right) t_{j}} \\
& +\sum_{(i, j) \in H_{2}} \frac{\left(\Delta_{2} x_{i, j}\right)\left(\Delta_{2} g\left(s_{i}, t_{j}\right)\right)}{s_{i}\left(\Delta t_{j}\right)}+\sum_{(i, j) \in H_{1,2}} \frac{\left(\Delta_{1} \Delta_{2} x_{i, j}\right)\left(\Delta_{1} \Delta_{2} g\left(s_{i}, t_{j}\right)\right)}{\left(\Delta s_{i}\right)\left(\Delta t_{j}\right)} .
\end{aligned}
$$

The MLE of $m$ based on $\left\{Z\left(s_{i}, t_{j}\right):(i, j) \in H\right\}$ has the form $\widetilde{m}_{H}=\zeta_{H} / A_{H}$ where

$$
\begin{aligned}
\zeta_{H}:= & \sum_{(i, j) \in H_{+}} \frac{g\left(s_{i}, t_{j}\right) Z\left(s_{i}, t_{j}\right)}{s_{i} t_{j}}-\sum_{(i, j) \in H_{-}} \frac{g\left(s_{i}, t_{j}\right) Z\left(s_{i}, t_{j}\right)}{s_{i} t_{j}} \\
& +\sum_{(i, j) \in H_{1}} \frac{\left(\Delta_{1} g\left(s_{i}, t_{j}\right)\right)\left(\Delta_{1} Z\left(s_{i}, t_{j}\right)\right)}{\left(\Delta s_{i}\right) t_{j}}+\sum_{(i, j) \in H_{2}} \frac{\left(\Delta_{2} g\left(s_{i}, t_{j}\right)\right)\left(\Delta_{2} Z\left(s_{i}, t_{j}\right)\right)}{s_{i}\left(\Delta t_{j}\right)} \\
& +\sum_{(i, j) \in H_{1,2}} \frac{\left(\Delta_{1} \Delta_{2} g\left(s_{i}, t_{j}\right)\right)\left(\Delta_{1} \Delta_{2} Z\left(s_{i}, t_{j}\right)\right)}{\left(\Delta s_{i}\right)\left(\Delta t_{j}\right)}
\end{aligned}
$$

and $\widetilde{m}_{H}$ has a normal distribution with mean $m$ and variance $1 / A_{H}$.

Proof Lemma 3.1 implies that the joint density of $\left\{Z\left(s_{i}, t_{j}\right):(i, j) \in H\right\}$ is

$$
c \cdot f\left(x_{i, j}-m g\left(s_{i}, t_{j}\right):(i, j) \in H_{+} \cup H_{-}\right) \cdot g\left(x_{i, j}-m g\left(s_{i}, t_{j}\right):(i, j) \in H\right) .
$$

Hence

$$
\begin{aligned}
& \frac{\mathrm{dP}_{Z}^{H}}{\mathrm{dP}_{W}^{H}}\left(x_{i, j}:(i, j) \in H\right)=\frac{f\left(x_{i, j}-m g\left(s_{i}, t_{j}\right):(i, j) \in H_{+} \cup H_{-}\right)}{f\left(x_{i, j}:(i, j) \in H_{+} \cup H_{-}\right)} \\
& \quad \times \frac{g\left(x_{i, j}-m g\left(s_{i}, t_{j}\right):(i, j) \in H\right)}{g\left(x_{i, j}:(i, j) \in H\right)}=\exp \left\{-\frac{1}{2}\left(A_{H} m^{2}-2 y_{H} m\right)\right\},
\end{aligned}
$$

which implies the given form of the MLE of $m$. Obviously, $\mathbf{E} \widetilde{m}_{H}=m$. 
Finally, using the independence of the increments in the terms of $\zeta_{H}$ one can easily show that $\mathrm{D}^{2} \zeta_{H}=A_{H}$.

\section{Weighted $L^{2}$-Riemann integrals of the 'partial derivatives' of a Wiener sheet}

We define $L^{2}$-Riemann integrals of a product of a function and a 'partial derivative' of an $L^{2}$-process along a curve $\Gamma=\{(s, \gamma(s)): s \in[a, b]\}$.

DeFinition 4.1 Let $Z$ be an $L^{2}$-process given along an $\varepsilon$-neighborhood of a curve $\Gamma:=\{(s, \gamma(s)): s \in[a, b]\}$, where $\gamma:[a, b] \rightarrow \mathbb{R}$ is strictly monotone. Consider a function $y:[a, b] \rightarrow \mathbb{R}$. Then

$$
\int_{a}^{b} y(s) Z(\mathrm{~d} s, \gamma(s)):=\operatorname{lin}_{h \rightarrow 0} . \frac{1}{h} \int_{a}^{b} y(s)[Z(s+h, \gamma(s))-Z(s, \gamma(s))] \mathrm{d} s,
$$

$$
\begin{aligned}
& \int_{\gamma(a)}^{\gamma(b)} y\left(\gamma^{-1}(t)\right) Z\left(\gamma^{-1}(t), \mathrm{d} t\right):=\operatorname{lin.m.} . \frac{1}{h} \int_{\gamma(a)}^{\gamma(b)} y\left(\gamma^{-1}(t)\right) \\
& \quad \times\left[Z\left(\gamma^{-1}(t), t+h\right)-Z\left(\gamma^{-1}(t), t\right)\right] \mathrm{d} t,
\end{aligned}
$$

if the right hand sides exist.

This definition is compatible with the notion of a weighted $L^{2}$-Riemann integral of the normal derivative of an $L^{2}$-process along a curve, see, for instance [12]. A weighted $L^{2}$-Riemann integral of the normal derivative along a curve can be expressed by the help of weighted $L^{2}$-Riemann integrals of the partial derivatives, namely

$$
\int_{\Gamma} y \partial_{\mathrm{n}} Z=-\int_{a}^{b} \gamma^{\prime}(s) y(s) Z(\mathrm{~d} s, \gamma(s))+\int_{\gamma(a)}^{\gamma(b)} \frac{y\left(\gamma^{-1}(t)\right)}{\gamma^{\prime}\left(\gamma^{-1}(t)\right)} Z\left(\gamma^{-1}(t), \mathrm{d} t\right) .
$$

However, we have to use weighted $L^{2}$-Riemann integrals of the partial derivatives, since the random variable $\zeta$ in Theorem 2.1 cannot be expressed only by the help of weighted $L^{2}$-Riemann integrals of the Wiener sheet and of its normal derivative. Note that by Lemma 3.5 of [12],

$$
\int_{\gamma(a)}^{\gamma(b)} y\left(\gamma^{-1}(t)\right) Z\left(\gamma^{-1}(t), \mathrm{d} t\right)=\underset{h \rightarrow 0}{\lim . \mathrm{m}} \frac{1}{h} \int_{a}^{b} \gamma^{\prime}(s) y(s)[Z(s, \gamma(s)+h)-Z(s, \gamma(s))] \mathrm{d} s,
$$


whenever $\gamma$ is differentiable on $(a, b)$ and the improper integrals on the right hand side exist. Hence a notation

$$
\int_{\gamma(a)}^{\gamma(b)} y\left(\gamma^{-1}(t)\right) Z\left(\gamma^{-1}(t), \mathrm{d} t\right)=\int_{a}^{b} \gamma^{\prime}(s) y(s) \partial_{2} Z(s, \gamma(s)) \mathrm{d} s
$$

would also be justified.

Next we give sufficient conditions for the existence of the integrals $\int_{a}^{b} y(s) W(\mathrm{~d} s, \gamma(s))$ and $\int_{\gamma(a)}^{\gamma(b)} y\left(\gamma^{-1}(t)\right) W\left(\gamma^{-1}(t), \mathrm{d} t\right)$, and we present approximating sums.

Proposition 4.2 Let $[a, b] \subset \mathbb{R}_{+}$, let $\gamma:[a, b] \rightarrow \mathbb{R}_{+}$and $y:[a, b] \rightarrow \mathbb{R}$ be continuous functions, and let $\gamma$ be strictly monotone. Then the integrals $\int_{a}^{b} y(s) W(\mathrm{~d} s, \gamma(s))$ and $\int_{\gamma(a)}^{\gamma(b)} y\left(\gamma^{-1}(t)\right) W\left(\gamma^{-1}(t), \mathrm{d} t\right)$ exist and

$$
\begin{aligned}
\int_{a}^{b} y(s) W(\mathrm{~d} s, \gamma(s)) & =\underset{N \rightarrow \infty}{\operatorname{i.m} .} S_{N}, \\
\int_{\gamma(a)}^{\gamma(b)} y\left(\gamma^{-1}(t)\right) W\left(\gamma^{-1}(t), \mathrm{d} t\right) & =\underset{N \rightarrow \infty}{\lim . \mathrm{m} .} \widetilde{S}_{N},
\end{aligned}
$$

where

$$
\begin{aligned}
S_{N} & :=\sum_{i=1}^{N-1} y\left(s_{i}^{(N)}\right)\left[W\left(s_{i+1}^{(N)}, \gamma\left(s_{i}^{(N)}\right)\right)-W\left(s_{i}^{(N)}, \gamma\left(s_{i}^{(N)}\right)\right)\right] \\
\widetilde{S}_{N} & :=\sum_{i=2}^{N} y\left(s_{i}^{(N)}\right)\left[W\left(s_{i}^{(N)}, \gamma\left(s_{i}^{(N)}\right)\right)-W\left(s_{i}^{(N)}, \gamma\left(s_{i-1}^{(N)}\right)\right)\right]
\end{aligned}
$$

and $\mathcal{P}_{N}: a=s_{1}^{(N)}<s_{2}^{(N)}<\cdots<s_{N-1}^{(N)}<s_{N}^{(N)}=b, \quad N=1,2, \ldots$, is a sequence of partitions such that $\left|\mathcal{P}_{N}\right| \rightarrow 0$ as $N \rightarrow \infty$.

Proof The existence of the limit $\lim _{N \rightarrow \infty} S_{N}$ follows from $\lim _{N, M \rightarrow \infty} \mathrm{E} S_{N} S_{M}=$ $\int_{a}^{b} \gamma(s) y(s)^{2} \mathrm{~d} s$, which is a consequence of Lemma 4.3 of [12]. In order to prove the first statement, we will show that for an arbitrary sequence $h_{N} \rightarrow 0$, we 
have $\lim _{N \rightarrow \infty} \mathrm{E}\left(S_{N}-I_{h_{N}}\right)^{2}=0$, where

$$
I_{h}:=\frac{1}{h} \int_{a}^{b} y(s)[Z(s+h, \gamma(s))-Z(s, \gamma(s))] \mathrm{d} s, \quad h \in \mathbb{R}, \quad h \neq 0 .
$$

Again by Lemma 4.3 of [12],

$$
\lim _{N \rightarrow \infty} \mathrm{E} S_{N}^{2}=\lim _{N \rightarrow \infty} \mathrm{E} I_{h_{N}}^{2}=\lim _{N \rightarrow \infty} \mathrm{E} S_{N} I_{h_{N}}=\int_{a}^{b} \gamma(s) y(s)^{2} \mathrm{~d} s,
$$

which completes the proof of the first statement.

Next suppose that function $\gamma$ is strictly increasing. Let $t_{i}^{(N)}:=\gamma\left(s_{i}^{(N)}\right)$, $i=1, \ldots, N$. Then $\widetilde{\mathcal{P}}_{N}: \gamma(a)=t_{1}^{(N)}<t_{2}^{(N)}<\cdots<t_{N-1}^{(N)}<t_{N}^{(N)}=\gamma(b)$, $N=1,2, \ldots$, is a sequence of partitions such that $\left|\widetilde{\mathcal{P}}_{N}\right| \rightarrow 0$ as $N \rightarrow \infty$ by the continuity of $\gamma$. Moreover,

$$
\widetilde{S}_{N}=\sum_{i=2}^{N} y\left(\gamma^{-1}\left(t_{i}^{(N)}\right)\right)\left[W\left(\gamma^{-1}\left(t_{i}^{(N)}\right), t_{i}^{(N)}\right)-W\left(\gamma^{-1}\left(t_{i}^{(N)}\right), t_{i-1}^{(N)}\right)\right],
$$

$N=1,2, \ldots$ Now, the arguments of the proof of the first statement imply the second statement.

Proposition 4.3 Let $[a, b] \subset \mathbb{R}_{+}$and let $\gamma:[a, b] \rightarrow \mathbb{R}_{+}$be a continuous strictly monotone function. Let $z$ be a real function given along an $\varepsilon$-neighborhood of the curve $\Gamma:=\{(s, \gamma(s)): s \in[a, b]\}$. Let

$$
\begin{gathered}
S_{N}:=\sum_{i=1}^{N-1}\left[z\left(s_{i+1}^{(N)}, \gamma\left(s_{i}^{(N)}\right)\right) W\left(s_{i+1}^{(N)}, \gamma\left(s_{i}^{(N)}\right)\right)-z\left(s_{i}^{(N)}, \gamma\left(s_{i}^{(N)}\right)\right) W\left(s_{i}^{(N)}, \gamma\left(s_{i}^{(N)}\right)\right)\right], \\
T_{N}:=\sum_{i=1}^{N-1} \frac{\left[z\left(s_{i+1}^{(N)}, \gamma\left(s_{i}^{(N)}\right)\right)-z\left(s_{i}^{(N)}, \gamma\left(s_{i}^{(N)}\right)\right)\right]}{s_{i+1}^{(N)}-s_{i}^{(N)}} \\
\quad \times\left[W\left(s_{i+1}^{(N)}, \gamma\left(s_{i}^{(N)}\right)\right)-W\left(s_{i}^{(N)}, \gamma\left(s_{i}^{(N)}\right)\right)\right] \\
U_{N}:=\sum_{i=1}^{N-1} \frac{\left[z\left(s_{i+1}^{(N)}, \gamma\left(s_{i+1}^{(N)}\right)\right)-z\left(s_{i}^{(N)}, \gamma\left(s_{i+1}^{(N)}\right)\right)\right]}{s_{i+1}^{(N)}-s_{i}^{(N)}} \\
\quad \times\left[W\left(s_{i+1}^{(N)}, \gamma\left(s_{i+1}^{(N)}\right)\right)-W\left(s_{i}^{(N)}, \gamma\left(s_{i+1}^{(N)}\right)\right)\right]
\end{gathered}
$$




$$
\begin{aligned}
& V_{N}:=\sum_{i=2}^{N} \frac{\left[z\left(s_{i}^{(N)}, \gamma\left(s_{i}^{(N)}\right)\right)-z\left(s_{i}^{(N)}, \gamma\left(s_{i-1}^{(N)}\right)\right)\right]}{\gamma\left(s_{i}^{(N)}\right)-\gamma\left(s_{i-1}^{(N)}\right)} \\
& \quad \times\left[W\left(s_{i}^{(N)}, \gamma\left(s_{i}^{(N)}\right)\right)-W\left(s_{i}^{(N)}, \gamma\left(s_{i-1}^{(N)}\right)\right)\right] .
\end{aligned}
$$

If $z$ and $\partial_{1} z$ are continuous then

$$
\begin{aligned}
& \underset{N \rightarrow \infty}{\lim .} S_{N}=\int_{a}^{b} z(s, \gamma(s)) W(\mathrm{~d} s, \gamma(s))+\int_{a}^{b} W(s, \gamma(s)) \partial_{1} z(s, \gamma(s)) \mathrm{d} s, \\
& \underset{N \rightarrow \infty}{\lim . T_{N}}=T_{N \rightarrow \infty} \lim _{N} U_{N}=\int_{a}^{b} \partial_{1} z(s, \gamma(s)) W(\mathrm{~d} s, \gamma(s)) .
\end{aligned}
$$

If $z$ and $\partial_{2} z$ are continuous then

$$
\underset{N \rightarrow \infty}{\lim .} V_{N}=\int_{\gamma(a)}^{\gamma(b)} \partial_{2} z\left(\gamma^{-1}(t), t\right) W\left(\gamma^{-1}(t), \mathrm{d} t\right) .
$$

Proof Consider the decomposition $S_{N}=S_{N}^{(1)}+S_{N}^{(2)}+S_{N}^{(3)}+S_{N}^{(4)}$, where

$$
\begin{aligned}
S_{N}^{(1)}:= & \sum_{i=1}^{N-1} z\left(s_{i}^{(N)}, \gamma\left(s_{i}^{(N)}\right)\right)\left[W\left(s_{i+1}^{(N)}, \gamma\left(s_{i}^{(N)}\right)\right)-W\left(s_{i}^{(N)}, \gamma\left(s_{i}^{(N)}\right)\right)\right] \\
S_{N}^{(2)}:= & \sum_{i=1}^{N-1} W\left(s_{i}^{(N)}, \gamma\left(s_{i}^{(N)}\right)\right)\left[\partial_{1} z\left(s_{i}^{(N)}, \gamma\left(s_{i}^{(N)}\right)\right)\right]\left(s_{i+1}^{(N)}-s_{i}^{(N)}\right), \\
S_{N}^{(3)}:= & \sum_{i=1}^{N-1}\left[z\left(s_{i+1}^{(N)}, \gamma\left(s_{i}^{(N)}\right)\right)-z\left(s_{i}^{(N)}, \gamma\left(s_{i}^{(N)}\right)\right)\right] \\
& \times\left[W\left(s_{i+1}^{(N)}, \gamma\left(s_{i}^{(N)}\right)\right)-W\left(s_{i}^{(N)}, \gamma\left(s_{i}^{(N)}\right)\right)\right] \\
S_{N}^{(4)}:= & \sum_{i=1}^{N-1}\left[z\left(s_{i+1}^{(N)}, \gamma\left(s_{i}^{(N)}\right)\right)-z\left(s_{i}^{(N)}, \gamma\left(s_{i}^{(N)}\right)\right)-\left(s_{i+1}^{(N)}-s_{i}^{(N)}\right) \partial_{1} z\left(s_{i}^{(N)}, \gamma\left(s_{i}^{(N)}\right)\right)\right] \\
& \times W\left(s_{i}^{(N)}, \gamma\left(s_{i}^{(N)}\right)\right) .
\end{aligned}
$$

By Proposition 4.2 we have $S_{N}^{(1)} \stackrel{\mathrm{L}_{2}}{\longrightarrow} \int_{a}^{b} z(s, \gamma(s)) W(\mathrm{~d} s, \gamma(s))$ and $S_{N}^{(2)} \stackrel{\mathrm{L}_{2}}{\longrightarrow}$ 
$\int_{a}^{b} W(s, \gamma(s)) \partial_{1} z(s, \gamma(s)) \mathrm{d} s$ as $N \rightarrow \infty$. Moreover, $S_{N}^{(3)} \stackrel{\mathrm{L}_{2}}{\longrightarrow} 0$ and $S_{N}^{(4)} \stackrel{\mathrm{L}_{2}}{\longrightarrow}$ 0 . Indeed,

$$
\begin{aligned}
\left\|S_{N}^{(3)}\right\|_{L^{2}}^{2} & \leq \sum_{i=1}^{N-1}\left|z\left(s_{i+1}^{(N)}, \gamma\left(s_{i}^{(N)}\right)\right)-z\left(s_{i}^{(N)}, \gamma\left(s_{i}^{(N)}\right)\right)\right|^{2} \gamma\left(s_{i}^{(N)}\right)\left(s_{i+1}^{(N)}-s_{i}^{(N)}\right) \\
& \leq(b-a) \sup _{\substack{s, \widetilde{s} \in[a, b] \\
|s-\widetilde{s}| \leqslant\left|\mathcal{P}_{N}\right|}} \gamma(s)|z(\widetilde{s}, \gamma(s))-z(s, \gamma(s))|^{2} \rightarrow 0
\end{aligned}
$$

as $N \rightarrow \infty$. Further, by the mean value theorem we have $z\left(s_{i+1}^{(N)}, \gamma\left(s_{i}^{(N)}\right)\right)-$ $z\left(s_{i}^{(N)}, \gamma\left(s_{i}^{(N)}\right)\right)=\partial_{1} z\left(\widetilde{s}_{i}^{(N)}, \gamma\left(s_{i}^{(N)}\right)\right)\left(s_{i+1}^{(N)}-s_{i}^{(N)}\right) \quad$ with some $\quad \widetilde{s}_{i}^{(N)} \in$ $\left(s_{i}^{(N)}, s_{i+1}^{(N)}\right)$, hence

$$
\begin{aligned}
\left\|S_{N}^{(4)}\right\|_{L^{2}} \leq & \sum_{i=1}^{N-1}\left|\partial_{1} z\left(\widetilde{s}_{i}^{(N)}, \gamma\left(s_{i}^{(N)}\right)\right)-\partial_{1} z\left(s_{i}^{(N)}, \gamma\left(s_{i}^{(N)}\right)\right)\right| \\
& \times\left(s_{i+1}^{(N)}-s_{i}^{(N)}\right)\left(s_{i}^{(N)} \gamma\left(s_{i}^{(N)}\right)\right)^{1 / 2} \\
\leq & (b-a) b^{1 / 2} \sup _{\substack{s, \widetilde{s} \in[a, b] \\
|s-\widetilde{s}| \leqslant\left|\mathcal{P}_{N}\right|}} \gamma(s)^{1 / 2}\left|\partial_{1} z(\widetilde{s}, \gamma(s))-\partial_{1} z(s, \gamma(s))\right| \rightarrow 0
\end{aligned}
$$

as $N \rightarrow \infty$.

Next consider the decomposition $T_{N}=T_{N}^{(1)}+T_{N}^{(2)}$, where

$$
\begin{aligned}
& T_{N}^{(1)}:=\sum_{i=1}^{N-1} {\left[\partial_{1} z\left(s_{i}^{(N)}, \gamma\left(s_{i}^{(N)}\right)\right)\right]\left[W\left(s_{i+1}^{(N)}, \gamma\left(s_{i}^{(N)}\right)\right)-W\left(s_{i}^{(N)}, \gamma\left(s_{i}^{(N)}\right)\right)\right] } \\
& T_{N}^{(2)}:=\sum_{i=1}^{N-1}\left[z\left(s_{i+1}^{(N)}, \gamma\left(s_{i}^{(N)}\right)\right)-z\left(s_{i}^{(N)}, \gamma\left(s_{i}^{(N)}\right)\right)-\left(s_{i+1}^{(N)}-s_{i}^{(N)}\right) \partial_{1} z\left(s_{i}^{(N)}, \gamma\left(s_{i}^{(N)}\right)\right)\right] \\
& \times\left[W\left(s_{i+1}^{(N)}, \gamma\left(s_{i}^{(N)}\right)\right)-W\left(s_{i}^{(N)}, \gamma\left(s_{i}^{(N)}\right)\right)\right] /\left(s_{i+1}^{(N)}-s_{i}^{(N)}\right) .
\end{aligned}
$$

By Proposition 4.2 we have $T_{N}^{(1)} \stackrel{\mathrm{L}_{2}}{\longrightarrow} \int_{a}^{b} \partial_{1} z(s, \gamma(s)) W(\mathrm{~d} s, \gamma(s))$ as $\quad N \rightarrow$ $\infty$. Moreover, $T_{N}^{(2)} \stackrel{\mathrm{L}_{2}}{\longrightarrow} 0$. Indeed, again by the mean value theorem, $z\left(s_{i+1}^{(N)}, \gamma\left(s_{i}^{(N)}\right)\right)-z\left(s_{i}^{(N)}, \gamma\left(s_{i}^{(N)}\right)\right)=\partial_{1} z\left(\widetilde{s}_{i}^{(N)}, \gamma\left(s_{i}^{(N)}\right)\right)\left(s_{i+1}^{(N)}-s_{i}^{(N)}\right)$ with some 
$\widetilde{s}_{i}^{(N)} \in\left(s_{i}^{(N)}, s_{i+1}^{(N)}\right)$, hence

$$
\begin{aligned}
\left\|T_{N}^{(2)}\right\|_{L^{2}}^{2} & \leq \sum_{i=1}^{N-1}\left|\partial_{1} z\left(\widetilde{s}_{i}^{(N)}, \gamma\left(s_{i}^{(N)}\right)\right)-\partial_{1} z\left(s_{i}^{(N)}, \gamma\left(s_{i}^{(N)}\right)\right)\right|^{2} \gamma\left(s_{i}^{(N)}\right)\left(s_{i+1}^{(N)}-s_{i}^{(N)}\right) \\
& \leq(b-a) \sup _{\substack{s, \widetilde{s} \in[a, b] \\
|s-\widetilde{s}| \leqslant\left|\mathcal{P}_{N}\right|}} \gamma(s)\left|\partial_{1} z(\widetilde{s}, \gamma(s))-\partial_{1} z(s, \gamma(s))\right|^{2} \rightarrow 0
\end{aligned}
$$

as $N \rightarrow \infty$. In a similar way one can prove $U_{N} \stackrel{\mathrm{L}_{2}}{\longrightarrow} \int_{a}^{b} \partial_{1} z(s, \gamma(s)) W(\mathrm{~d} s, \gamma(s))$ as $N \rightarrow \infty$ using $\sum_{i=1}^{N-1} y\left(s_{i+1}^{(N)}\right)\left[W\left(s_{i+1}^{(N)}, \gamma\left(s_{i+1}^{(N)}\right)\right)-W\left(s_{i}^{(N)}, \gamma\left(s_{i+1}^{(N)}\right)\right)\right] \stackrel{\mathrm{L}_{2}}{\longrightarrow}$ $\int_{a}^{b} y(s) W(\mathrm{~d} s, \gamma(s))$ as $N \rightarrow \infty$ for a continuous function $y:[a, b] \rightarrow \mathbb{R}$, which can be shown as the first statement in Proposition 4.2 , with a slight suitable modification of Lemma 4.3 of [12]. The last statement can be proved by similar arguments.

\section{$5 \quad$ Proof of Theorem 2.1}

In order to determine Radon-Nikodym derivatives we have developed the following general method based on the results of [14, Section 2.3.2] (see e.g. $[12,13])$. Let $X$ be a separable metric space. For a random field $\left\{\xi_{x}: x \in X\right\}$ with continuous trajectories on a probability space $(\Omega, \mathcal{A}, \mathrm{P})$, the induced mapping $\xi: \Omega \rightarrow C(X)$ is measurable. Let $\mathrm{P}_{\xi}$ denote the probability measure generated by the process $\xi$ on $(C(X), \mathcal{B}(C(X)))$. For a finite set $X^{\prime}=\left\{x_{1}, \ldots, x_{k}\right\} \subset X$, we denote by $\mathrm{P}_{\xi}^{X^{\prime}}$ the probability measure generated by the random variable $\xi\left(X^{\prime}\right):=\left(\xi_{x_{1}}, \ldots, \xi_{x_{k}}\right)$ on $\left(\mathbb{R}^{k}, \mathcal{B}\left(\mathbb{R}^{k}\right)\right)$.

Proposition 5.1 Let $\left\{\xi_{x}: x \in X\right\}$ and $\left\{\widetilde{\xi}_{x}: x \in X\right\}$ be random fields with continuous trajectories. Suppose that there exists a measurable function $f: C(X) \rightarrow \mathbb{R}$ such that $\mathrm{E} f(\xi)=1$ and that for any finite set $X_{0} \subset X$, there exists a sequence of finite subsets $X_{n}, n=1,2, \ldots$ with $X_{0} \subset X_{n} \subset X$, $n=1,2, \ldots$, and with

$$
\frac{\mathrm{dP}_{\widetilde{\xi}}^{X_{n}}}{\mathrm{dP}_{\xi}^{X_{n}}}\left(\xi\left(X_{n}\right)\right) \stackrel{\mathrm{P}}{\longrightarrow} f(\xi) \quad \text { as } n \rightarrow \infty
$$

Then $\mathrm{P}_{\widetilde{\xi}}$ is absolutely continuous with respect to $\mathrm{P}_{\xi}$ and $\frac{\mathrm{dP}_{\widetilde{\xi}}}{\mathrm{dP}_{\xi}}=f$.

(The proof can be found in [13].) 


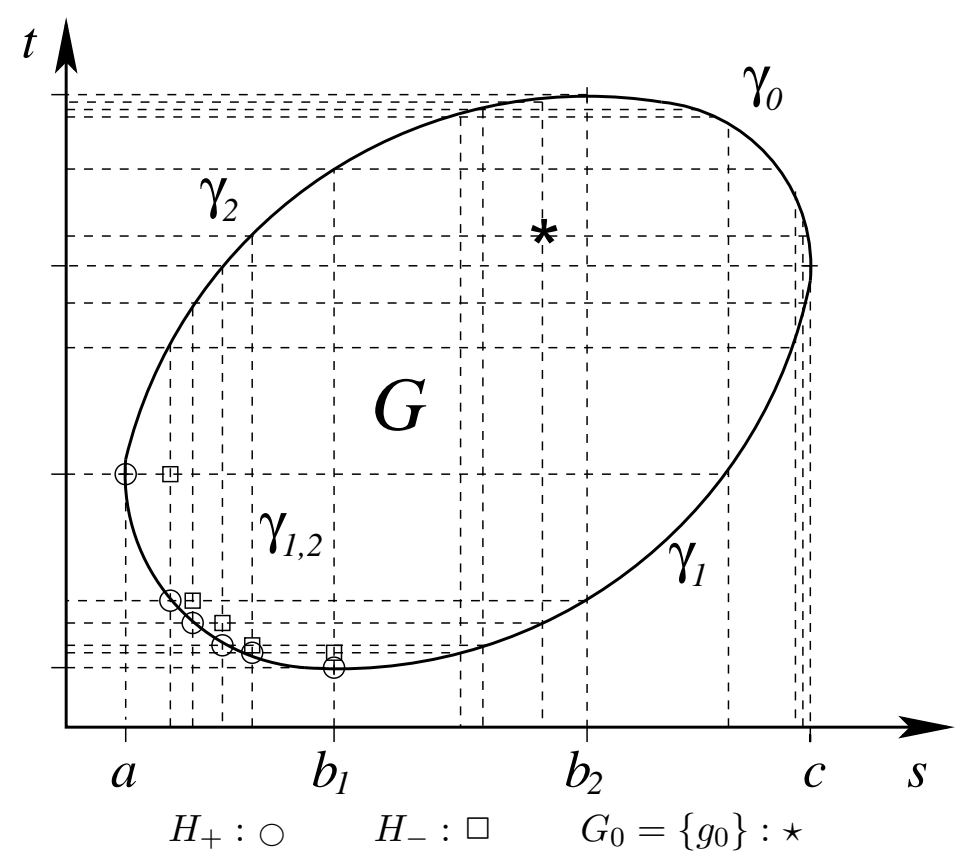

Figure 3. A example of a grid on $[a, c] \times\left[\gamma_{1}\left(b_{1}\right), \gamma_{2}\left(b_{2}\right)\right]$ containing a one point set $G_{0}$.

We will apply Proposition 5.1 for $X=G$ and for the random fields $\xi=W$ and $\widetilde{\xi}=Z$. Observe, that $\zeta$ can also be expressed in integrals with respect to the Wiener sheet $\{W(s, t): s, t \geq 0\}$, namely $\zeta=\eta+m A$, where

$$
\begin{aligned}
\eta:= & \frac{g\left(b_{1}, \gamma_{1,2}\left(b_{1}\right)\right) W\left(b_{1}, \gamma_{1,2}\left(b_{1}\right)\right)}{b_{1} \gamma_{1,2}\left(b_{1}\right)}+\int_{b_{1}}^{c} \frac{\partial_{1} g\left(s, \gamma_{1}(s)\right)}{\gamma_{1}(s)} W\left(\mathrm{~d} s, \gamma_{1}(s)\right) \\
& +\int_{a}^{b_{1}} \frac{\left[g \left(s, \gamma_{1,2}(s)-s \partial_{1} g\left(s, \gamma_{1,2}(s)\right]\right.\right.}{s^{2} \gamma_{1,2}(s)}\left[W\left(s, \gamma_{1,2}(s)\right) \mathrm{d} s-s W\left(\mathrm{~d} s, \gamma_{1,2}(s)\right)\right] \\
& +\int_{\gamma_{1,2}\left(b_{1}\right)}^{\gamma_{1,2}(a)} \frac{\partial_{2} g\left(\gamma_{1,2}^{-1}(t), t\right)}{\gamma_{1,2}^{-1}(t)} W\left(\gamma_{1,2}^{-1}(t), \mathrm{d} t\right)+\int_{\gamma_{2}(a)}^{\gamma_{2}\left(b_{2}\right)} \frac{\partial_{2} g\left(\gamma_{2}^{-1}(t), t\right)}{\gamma_{2}^{-1}(t)} W\left(\gamma_{2}^{-1}(t), \mathrm{d} t\right) \\
& +\iint_{G} \partial_{1} \partial_{2} g(s, t) W(\mathrm{~d} s, \mathrm{~d} t) .
\end{aligned}
$$

Let us consider a finite subset $G_{0} \subset G$. Condition (2.1) ensures that one 
can construct a grid on the rectangle $[a, c] \times\left[\gamma_{1}\left(b_{1}\right), \gamma_{2}\left(b_{2}\right)\right]$ containing $G_{0}$ such that the grid points on the boundaries $\Gamma_{1,2}, \Gamma_{1}$ and $\Gamma_{2}$ have the forms $\left(s, \gamma_{1,2}(s)\right),\left(s, \gamma_{1}(s)\right)$ and $\left(s, \gamma_{2}(s)\right)$, respectively. More precisely, there exist $k, m \in \mathbb{N}, \quad \ell \in \mathbb{N} \cup\{0\}$ and real numbers

$$
\begin{aligned}
a=s_{1}<\cdots<s_{k} & =b_{1} \wedge b_{2}<s_{k+1}<\cdots<s_{k+\ell} \\
& =b_{1} \vee b_{2}<s_{k+\ell+1}<\cdots<s_{k+\ell+m}=c
\end{aligned}
$$

such that the grid $\left(s_{i}, t_{j}\right), i=1, \ldots, k+\ell+m, j=1, \ldots, 2 k+\ell$ with

$$
t_{i}:=\gamma_{1,2}\left(s_{r-i+1}\right), \quad i=1, \ldots, r, \quad t_{r+i}:=\gamma_{2}\left(s_{i}\right), \quad i=1, \ldots, 2 k+\ell-r
$$

satisfies

$$
\gamma_{1}\left(s_{i}\right)=t_{i-r} \quad \text { for } \quad i=r+1, \ldots, k+\ell+m
$$

where

$$
r:= \begin{cases}k & \text { if } b_{1} \leq b_{2} \\ k+\ell & \text { if } b_{1}>b_{2}\end{cases}
$$

Figure 3 shows an example of an appropriate grid for $G_{0}$ containing a single point $g_{0} \in G$.

Now, using the notations of Section 3 let

$$
H:=\left\{(i, j) \in \mathbb{N}^{2}: 1 \leq i \leq k+\ell+m, 1 \leq j \leq 2 k+\ell,\left(s_{i}, t_{j}\right) \in G\right\} .
$$

From the special form of the grid follows that

$$
\begin{aligned}
H_{+} & =\{(i, r-i+1): i=1, \ldots, r\}, \quad H_{-}=\{(i, r-i+2): i=2, \ldots, r\}, \\
H_{1} & =H_{-} \cup\{(i, i-r): i=r+1, \ldots, k+\ell+m\}, \\
H_{2} & =H_{-} \cup\{(i, r+i): i=1, \ldots, 2 k+\ell-r\} .
\end{aligned}
$$

Using Lemma 3.2 we conclude, that

$$
\frac{\mathrm{dP}_{Z}^{H}}{\mathrm{dP}_{W}^{H}}\left(W\left(s_{i}, t_{j}\right):(i, j) \in H\right)=\exp \left\{-\frac{1}{2}\left(A_{H} m^{2}-2 \eta_{H} m\right)\right\},
$$


where

$$
\begin{aligned}
A_{H}=\sum_{i=1}^{r} \frac{g\left(s_{i}, \gamma_{1,2}\left(s_{i}\right)\right)^{2}}{s_{i} \gamma_{1,2}\left(s_{i}\right)} & -\sum_{i=2}^{r} \frac{g\left(s_{i}, \gamma_{1,2}\left(s_{i-1}\right)\right)^{2}}{s_{i} \gamma_{1,2}\left(s_{i-1}\right)}+\sum_{(i, j) \in H_{1,2}} \frac{\left(\Delta_{1} \Delta_{2} g\left(s_{i}, t_{j}\right)\right)^{2}}{\left(\Delta s_{i}\right)\left(\Delta t_{j}\right)} \\
& +\sum_{i=2}^{r} \frac{\left[g\left(s_{i}, \gamma_{1,2}\left(s_{i-1}\right)\right)-g\left(s_{i-1}, \gamma_{1,2}\left(s_{i-1}\right)\right]^{2}\right.}{\left(\Delta s_{i}\right) \gamma_{1,2}\left(s_{i-1}\right)} \\
& +\sum_{i=r+1}^{k+\ell+m} \frac{\left[g\left(s_{i}, \gamma_{1}\left(s_{i}\right)\right)-g\left(s_{i-1}, \gamma_{1}\left(s_{i}\right)\right]^{2}\right.}{\left(\Delta s_{i}\right) \gamma_{1}\left(s_{i}\right)} \\
& +\sum_{i=2}^{r} \frac{\left[g\left(s_{i}, \gamma_{1,2}\left(s_{i-1}\right)\right)-g\left(s_{i}, \gamma_{1,2}\left(s_{i}\right)\right)\right]^{2}}{s_{i}\left(\gamma_{1,2}\left(s_{i-1}\right)-\gamma_{1,2}\left(s_{i}\right)\right)} \\
& +\sum_{i=2}^{2 k+\ell-r} \frac{\left[g\left(s_{i}, \gamma_{2}\left(s_{i}\right)\right)-g\left(s_{i}, \gamma_{2}\left(s_{i-1}\right)\right)\right]^{2}}{s_{i}\left(\gamma_{2}\left(s_{i}\right)-\gamma_{2}\left(s_{i-1}\right)\right)}
\end{aligned}
$$

and

$$
\begin{aligned}
\eta_{H}= & \sum_{i=1}^{r} \frac{g\left(s_{i}, \gamma_{1,2}\left(s_{i}\right)\right) W\left(s_{i}, \gamma_{1,2}\left(s_{i}\right)\right)}{s_{i} \gamma_{1,2}\left(s_{i}\right)}-\sum_{i=2}^{r} \frac{g\left(s_{i}, \gamma_{1,2}\left(s_{i-1}\right)\right) W\left(s_{i}, \gamma_{1,2}\left(s_{i-1}\right)\right)}{s_{i} \gamma_{1,2}\left(s_{i-1}\right)} \\
& +\sum_{(i, j) \in H_{1,2}} \frac{\left(\Delta_{1} \Delta_{2} g\left(s_{i}, t_{j}\right)\right)\left(\Delta_{1} \Delta_{2} W\left(s_{i}, t_{j}\right)\right)}{\left(\Delta s_{i}\right)\left(\Delta t_{j}\right)} \\
& +\sum_{i=2}^{r} \frac{\left[g\left(s_{i}, \gamma_{1,2}\left(s_{i-1}\right)\right)-g\left(s_{i-1}, \gamma_{1,2}\left(s_{i-1}\right)\right)\right]}{\left(\Delta s_{i}\right) \gamma_{1,2}\left(s_{i-1}\right)} \\
& +\sum_{i=r+1}^{k+\ell+m} \frac{\left[g\left(s_{i}, \gamma_{1}\left(s_{i}\right)\right)-g\left(s_{i-1}, \gamma_{1}\left(s_{i}\right)\right)\right]\left[W\left(s_{i}, \gamma_{1}\left(s_{i}\right)\right)-W\left(s_{i-1}, \gamma_{1}\left(s_{i}\right)\right)\right]}{\left(\Delta s_{i}\right) \gamma_{1}\left(s_{i}\right)} \\
& +\sum_{i=2}^{r} \frac{\left[g\left(s_{i}, \gamma_{1,2}\left(s_{i-1}\right)\right)-g\left(s_{i}, \gamma_{1,2}\left(s_{i}\right)\right)\right]\left[W\left(s_{i}, \gamma_{1,2}\left(s_{i-1}\right)\right)-W\left(s_{i}, \gamma_{1,2}\left(s_{i}\right)\right)\right]}{s_{i}\left(\gamma_{1,2}\left(s_{i-1}\right)-\gamma_{1,2}\left(s_{i}\right)\right)} \\
& +\sum_{i=1}^{2 k+\ell-r} \frac{\left[g\left(s_{i}, \gamma_{2}\left(s_{i}\right)\right)-g\left(s_{i}, \gamma_{2}\left(s_{i-1}\right)\right)\right]\left[W\left(s_{i}, \gamma_{2}\left(s_{i}\right)\right)-W\left(s_{i}, \gamma_{2}\left(s_{i-1}\right)\right)\right]}{s_{i}\left(\gamma_{2}\left(s_{i}\right)-\gamma_{2}\left(s_{i-1}\right)\right)} .
\end{aligned}
$$

Hence, by Proposition 5.1, it is sufficient to prove that for any sequence of 
partitions

$$
\begin{aligned}
\mathcal{P}_{N}: a & =s_{1}^{(N)}<\cdots<s_{k_{N}}^{(N)}=b_{1} \wedge b_{2}<s_{k_{N}+1}^{(N)}<\cdots<s_{k_{N}+\ell_{N}}^{(N)} \\
& =b_{1} \vee b_{2}<s_{k_{N}+\ell_{N}+1}^{(N)}<\cdots<s_{k_{N}+\ell_{N}+m_{N}}^{(N)}=c,
\end{aligned}
$$

with $\left|\mathcal{P}_{N}\right| \rightarrow 0$, for $t_{j}^{(N)}, j=1, \ldots, 2 k_{N}+\ell_{N}, N=1,2, \ldots$, defined as in (5.1), and for

$$
\begin{aligned}
H_{1,2}^{(N)}:=\{(i, j): & 1 \leq i \leq k_{N}+\ell_{N}+m_{N} \text { and } 1 \leq j \leq 2 k_{N}+\ell_{N}-1 \\
& \text { and } \left.\left(s_{i}^{(N)}, t_{j}^{(N)}\right) \in G \backslash\left(\Gamma_{1,2} \cup \Gamma_{1} \cup \Gamma_{2}\right)\right\}, \quad N=1,2, \ldots,
\end{aligned}
$$

we have $A_{H_{N}} \rightarrow A$ and $\eta_{H_{N}} \stackrel{\mathrm{L}_{2}}{\longrightarrow} \eta$, where

$$
A_{H_{N}}=\sum_{i=1}^{6} A_{N}^{(i)}, \quad \eta_{H_{N}}=\sum_{i=1}^{6} \eta_{N}^{(i)}
$$

with

$$
\begin{aligned}
A_{N}^{(1)} & :=\sum_{i=1}^{r_{N}} \frac{g\left(s_{i}^{(N)}, \gamma_{1,2}\left(s_{i}^{(N)}\right)\right)^{2}}{s_{i}^{(N)} \gamma_{1,2}\left(s_{i}^{(N)}\right)}-\sum_{i=2}^{r_{N}} \frac{g\left(s_{i}^{(N)}, \gamma_{1,2}\left(s_{i-1}^{(N)}\right)\right)^{2}}{s_{i}^{(N)} \gamma_{1,2}\left(s_{i-1}^{(N)}\right)} \\
A_{N}^{(2)}: & =\sum_{i=2}^{r_{N}} \frac{\left[g\left(s_{i}^{(N)}, \gamma_{1,2}\left(s_{i-1}^{(N)}\right)\right)-g\left(s_{i-1}^{(N)}, \gamma_{1,2}\left(s_{i-1}^{(N)}\right)\right]^{2}\right.}{\left(\Delta s_{i}^{(N)}\right) \gamma_{1,2}\left(s_{i-1}^{(N)}\right)}, \\
A_{N}^{(3)}:= & \sum_{i=r_{N}+1}^{k_{N}+\ell_{N}+m_{N}} \frac{\left[g\left(s_{i}^{(N)}, \gamma_{1}\left(s_{i}^{(N)}\right)\right)-g\left(s_{i-1}^{(N)}, \gamma_{1}\left(s_{i}^{(N)}\right)\right]^{2}\right.}{\left(\Delta s_{i}^{(N)}\right) \gamma_{1}\left(s_{i}^{(N)}\right)}, \\
A_{N}^{(4)}:= & \sum_{i=2}^{r_{N}} \frac{\left[g\left(s_{i}^{(N)}, \gamma_{1,2}\left(s_{i-1}^{(N)}\right)\right)-g\left(s_{i}^{(N)}, \gamma_{1,2}\left(s_{i}^{(N)}\right)\right)\right]^{2}}{s_{i}^{(N)}\left(\gamma_{1,2}\left(s_{i-1}^{(N)}\right)-\gamma_{1,2}\left(s_{i}^{(N)}\right)\right)}, \\
A_{N}^{(5)}:= & \sum_{i=2} \frac{\left[g\left(s_{i}^{(N)}, \gamma_{2}\left(s_{i}^{(N)}\right)\right)-g\left(s_{i}^{(N)}, \gamma_{2}\left(s_{i-1}^{(N)}\right)\right)\right]^{2}}{s_{i}^{(N)}\left(\gamma_{2}\left(s_{i}^{(N)}\right)-\gamma_{2}\left(s_{i-1}^{(N)}\right)\right)} \\
A_{N}^{(6)}:= & \sum_{(i, j) \in H_{1,2}^{(N)}} \frac{\left(\Delta_{1} \Delta_{2} g\left(s_{i}^{(N)}, t_{j}^{(N)}\right)\right)^{2}}{\left(\Delta s_{i}^{(N)}\right)\left(\Delta t_{j}^{(N)}\right)},
\end{aligned}
$$


and

$$
\begin{aligned}
& \eta_{N}^{(1)}:=\sum_{i=1}^{r_{N}} \frac{g\left(s_{i}^{(N)}, \gamma_{1,2}\left(s_{i}^{(N)}\right)\right) W\left(s_{i}^{(N)}, \gamma_{1,2}\left(s_{i}^{(N)}\right)\right)}{s_{i}^{(N)} \gamma_{1,2}\left(s_{i}^{(N)}\right)} \\
& -\sum_{i=2}^{r_{N}} \frac{g\left(s_{i}^{(N)}, \gamma_{1,2}\left(s_{i-1}^{(N)}\right)\right) W\left(s_{i}^{(N)}, \gamma_{1,2}\left(s_{i-1}^{(N)}\right)\right)}{s_{i}^{(N)} \gamma_{1,2}\left(s_{i-1}^{(N)}\right)} \\
& \eta_{N}^{(2)}:=\sum_{i=2}^{r_{N}} \frac{\left[g\left(s_{i}^{(N)}, \gamma_{1,2}\left(s_{i-1}^{(N)}\right)\right)-g\left(s_{i-1}^{(N)}, \gamma_{1,2}\left(s_{i-1}^{(N)}\right)\right)\right]}{\left(\Delta s_{i}^{(N)}\right) \gamma_{1,2}\left(s_{i-1}^{(N)}\right)} \\
& \times\left[W\left(s_{i}^{(N)}, \gamma_{1,2}\left(s_{i-1}^{(N)}\right)\right)-W\left(s_{i-1}^{(N)}, \gamma_{1,2}\left(s_{i-1}^{(N)}\right)\right)\right], \\
& \eta_{N}^{(3)}:=\sum_{i=r_{N}+1}^{k_{N}+\ell_{N}+m_{N}} \frac{\left[g\left(s_{i}^{(N)}, \gamma_{1}\left(s_{i}^{(N)}\right)\right)-g\left(s_{i-1}^{(N)}, \gamma_{1}\left(s_{i}^{(N)}\right)\right)\right]}{\left(\Delta s_{i}^{(N)}\right) \gamma_{1}\left(s_{i}^{(N)}\right)} \\
& \times\left[W\left(s_{i}^{(N)}, \gamma_{1}\left(s_{i}^{(N)}\right)\right)-W\left(s_{i-1}^{(N)}, \gamma_{1}\left(s_{i}^{(N)}\right)\right)\right], \\
& \eta_{N}^{(4)}:=\sum_{i=2}^{r_{N}} \frac{\left[g\left(s_{i}^{(N)}, \gamma_{1,2}\left(s_{i-1}^{(N)}\right)\right)-g\left(s_{i}^{(N)}, \gamma_{1,2}\left(s_{i}^{(N)}\right)\right)\right]}{s_{i}^{(N)}\left(\gamma_{1,2}\left(s_{i-1}^{(N)}\right)-\gamma_{1,2}\left(s_{i}^{(N)}\right)\right)} \\
& \times\left[W\left(s_{i}^{(N)}, \gamma_{1,2}\left(s_{i-1}^{(N)}\right)\right)-W\left(s_{i}^{(N)}, \gamma_{1,2}\left(s_{i}^{(N)}\right)\right)\right], \\
& \eta_{N}^{(5)}:=\sum_{i=2}^{2 k_{N}+\ell_{N}-r_{N}} \frac{\left[g\left(s_{i}^{(N)}, \gamma_{2}\left(s_{i}^{(N)}\right)\right)-g\left(s_{i}^{(N)}, \gamma_{2}\left(s_{i-1}^{(N)}\right)\right)\right]}{s_{i}^{(N)}\left(\gamma_{2}\left(s_{i}^{(N)}\right)-\gamma_{2}\left(s_{i-1}^{(N)}\right)\right)} \\
& \times\left[W\left(s_{i}^{(N)}, \gamma_{2}\left(s_{i}^{(N)}\right)\right)-W\left(s_{i}^{(N)}, \gamma_{2}\left(s_{i-1}^{(N)}\right)\right)\right], \\
& \eta_{N}^{(6)}:=\sum_{(i, j) \in H_{1,2}^{(N)}} \frac{\left(\Delta_{1} \Delta_{2} g\left(s_{i}^{(N)}, t_{j}^{(N)}\right)\right)\left(\Delta_{1} \Delta_{2} W\left(s_{i}^{(N)}, t_{j}^{(N)}\right)\right)}{\left(\Delta s_{i}^{(N)}\right)\left(\Delta t_{j}^{(N)}\right)} .
\end{aligned}
$$

It is easy to see that $A_{N} \rightarrow A$ as $N \rightarrow \infty$ since

$$
\begin{aligned}
A_{N}^{(1)} & =\frac{g\left(b_{1}, \gamma_{1,2}\left(b_{1}\right)\right)^{2}}{b_{1} \gamma_{1,2}\left(b_{1}\right)}-\sum_{i=2}^{r_{N}} \frac{1}{\gamma_{1,2}\left(s_{i-1}^{(N)}\right)}\left[\frac{g\left(s_{i}^{(N)}, \gamma_{1,2}\left(s_{i-1}^{(N)}\right)\right)^{2}}{s_{i}^{(N)}}-\frac{g\left(s_{i-1}^{(N)}, \gamma_{1,2}\left(s_{i-1}^{(N)}\right)\right)^{2}}{s_{i-1}^{(N)}}\right] \\
& \rightarrow \frac{g\left(b_{1}, \gamma_{1,2}\left(b_{1}\right)\right)^{2}}{b_{1} \gamma_{1,2}\left(b_{1}\right)}+\int_{a}^{b_{1}} \frac{g\left(s, \gamma_{1,2}(s)\right)\left[g \left(s, \gamma_{1,2}(s)-2 s \partial_{1} g\left(s, \gamma_{1,2}(s)\right]\right.\right.}{s^{2} \gamma_{1,2}(s)} \mathrm{d} s
\end{aligned}
$$


$A_{N}^{(2)}+A_{N}^{(3)} \rightarrow \int_{a}^{b_{1}} \frac{\left[\partial_{1} g\left(s, \gamma_{1,2}(s)\right)\right]^{2}}{\gamma_{1,2}(s)} \mathrm{d} s+\int_{b_{1}}^{c} \frac{\left[\partial_{1} g\left(s, \gamma_{1}(s)\right)\right]^{2}}{\gamma_{1}(s)} \mathrm{d} s$,
$A_{N}^{(4)}+A_{N}^{(5)} \rightarrow \int_{\gamma_{1,2}\left(b_{1}\right)}^{\gamma_{1,2}(a)} \frac{\left[\partial_{2} g\left(\gamma_{1,2}^{-1}(t), t\right)\right]^{2}}{\gamma_{1,2}^{-1}(t)} \mathrm{d} t+\int_{\gamma_{2}(a)}^{\gamma_{2}\left(b_{2}\right)} \frac{\left[\partial_{2} g\left(\gamma_{2}^{-1}(t), t\right)\right]^{2}}{\gamma_{2}^{-1}(t)} \mathrm{d} t$,
$A_{N}^{(6)}=\sum_{(i, j) \in H_{1,2}^{(N)}}\left[\frac{\Delta_{1} \Delta_{2} g\left(s_{i}^{(N)}, t_{j}^{(N)}\right)}{\left(\Delta s_{i}^{(N)}\right)\left(\Delta t_{j}^{(N)}\right)}\right]^{2}\left(\Delta s_{i}^{(N)}\right)\left(\Delta t_{j}^{(N)}\right) \rightarrow \iint_{G}\left[\partial_{1} \partial_{2} g(s, t)\right]^{2} \mathrm{~d} s \mathrm{~d} t$.

The aim of the following discussion is to prove convergence $\eta_{N} \stackrel{\mathrm{L}_{2}}{\longrightarrow} \eta$ as $N \rightarrow \infty$ which completes the proof. We have

$$
\begin{aligned}
\eta_{N}^{(1)}= & \frac{g\left(b_{1}, \gamma_{1,2}\left(b_{1}\right)\right) W\left(b_{1}, \gamma_{1,2}\left(b_{1}\right)\right)}{b_{1} \gamma_{1,2}\left(b_{1}\right)} \\
-\sum_{i=2}^{r_{N}} \frac{1}{\gamma_{1,2}\left(s_{i-1}^{(N)}\right)}\left[\frac{g\left(s_{i}^{(N)}, \gamma_{1,2}\left(s_{i-1}^{(N)}\right)\right) W\left(s_{i}^{(N)}, \gamma_{1,2}\left(s_{i-1}^{(N)}\right)\right)}{s_{i}^{(N)}}\right. & \\
& \left.-\frac{g\left(s_{i-1}^{(N)}, \gamma_{1,2}\left(s_{i-1}^{(N)}\right)\right) W\left(s_{i-1}^{(N)}, \gamma_{1,2}\left(s_{i-1}^{(N)}\right)\right)}{s_{i-1}^{(N)}}\right],
\end{aligned}
$$

hence applying Proposition 4.3 for the curve $\gamma_{1,2}$ and for the function $(s, t) \mapsto$ $s^{-1} t^{-1} g(s, t)$, we obtain

$$
\begin{aligned}
\eta_{N}^{(1)} \stackrel{\mathrm{L}_{2}}{\longrightarrow} \frac{g\left(b_{1}, \gamma_{1,2}\left(b_{1}\right)\right) W\left(b_{1}, \gamma_{1,2}\left(b_{1}\right)\right)}{b_{1} \gamma_{1,2}\left(b_{1}\right)}-\int_{a}^{b_{1}} \frac{g\left(s, \gamma_{1,2}(s)\right)}{s \gamma_{1,2}(s)} W\left(\mathrm{~d} s, \gamma_{1,2}(s)\right) \\
-\int_{a}^{b_{1}} \frac{\left[s \partial_{1} g\left(s, \gamma_{1,2}(s)\right)-g\left(s, \gamma_{1,2}(s)\right)\right] W\left(s, \gamma_{1,2}(s)\right)}{s^{2} \gamma_{1,2}(s)} \mathrm{d} s
\end{aligned}
$$

Applying Proposition 4.3 we also conclude

$$
\eta_{N}^{(2)} \stackrel{\mathrm{L}_{2}}{\longrightarrow} \int_{a}^{b_{1}} \frac{\partial_{1} g\left(s, \gamma_{1,2}(s)\right)}{\gamma_{1,2}(s)} W\left(\mathrm{~d} s, \gamma_{1,2}(s)\right),
$$




$$
\begin{aligned}
& \eta_{N}^{(3)} \stackrel{\mathrm{L}_{2}}{\longrightarrow} \int_{b_{1}}^{c} \frac{\partial_{1} g\left(s, \gamma_{1}(s)\right)}{\gamma_{1}(s)} W\left(\mathrm{~d} s, \gamma_{1}(s)\right), \\
& \eta_{N}^{(4)} \stackrel{\mathrm{L}_{2}}{\longrightarrow} \int_{\gamma_{1,2}\left(b_{1}\right)}^{\gamma_{1,2}(a)} \frac{\partial_{2} g\left(\gamma_{1,2}^{-1}(t), t\right)}{\gamma_{1,2}^{-1}(t)} W\left(\gamma_{1,2}^{-1}(t), \mathrm{d} t\right), \\
& \eta_{N}^{(5)} \stackrel{\mathrm{L}_{2}}{\longrightarrow} \int_{\gamma_{2}(a)}^{\gamma_{2}\left(b_{2}\right)} \frac{\partial_{2} g\left(\gamma_{2}^{-1}(t), t\right)}{\gamma_{2}^{-1}(t)} W\left(\gamma_{2}^{-1}(t), \mathrm{d} t\right) .
\end{aligned}
$$

Finally, consider the decomposition $\eta_{N}^{(6)}=\eta_{N}^{(6, A)}+\eta_{N}^{(6, B)}$, where

$$
\begin{aligned}
\eta_{N}^{(6, A)}:= & \sum_{(i, j) \in H_{1,2}^{(N)}}\left[\partial_{1} \partial_{2} g\left(s_{i}^{(N)}, t_{j}^{(N)}\right)\right]\left[\Delta_{1} \Delta_{2} W\left(s_{i}^{(N)}, t_{j}^{(N)}\right)\right] \\
\eta_{N}^{(6, B)}:= & \sum_{(i, j) \in H_{1,2}^{(N)}} \frac{\left[\Delta_{1} \Delta_{2} g\left(s_{i}^{(N)}, t_{j}^{(N)}\right)-\left(\Delta s_{i}^{(N)}\right)\left(\Delta t_{j}^{(N)}\right) \partial_{1} \partial_{2} g\left(s_{i}^{(N)}, t_{j}^{(N)}\right)\right]}{\left(\Delta s_{i}^{(N)}\right)\left(\Delta t_{j}^{(N)}\right)} \\
& \times\left[\Delta_{1} \Delta_{2} W\left(s_{i}^{(N)}, t_{j}^{(N)}\right)\right] .
\end{aligned}
$$

Clearly $\eta_{N}^{(6, A)} \stackrel{\mathrm{L}_{2}}{\longrightarrow} \iint_{G} \partial_{1} \partial_{2} g(s, t) W(\mathrm{~d} s, \mathrm{~d} t)$ as $N \rightarrow \infty$. Moreover $\eta_{N}^{(6, B)} \stackrel{\mathrm{L}_{2}}{\longrightarrow}$ $0 \quad$ as $N \rightarrow \infty$. Indeed, by the mean value theorem, $\Delta_{1} \Delta_{2} g\left(s_{i}^{(N)}, t_{j}^{(N)}\right)=$ $\left(\Delta s_{i}^{(N)}\right)\left(\Delta t_{j}^{(N)}\right) \partial_{1} \partial_{2} g\left(\widetilde{s}_{i}^{(N)}, \widetilde{t}_{j}^{(N)}\right)$ with some $\widetilde{s}_{i}^{(N)} \in\left(s_{i-1}^{(N)}, s_{i}^{(N)}\right)$ and $\widetilde{t}_{j}^{(N)} \in$ $\left(t_{j-1}^{(N)}, t_{j}^{(N)}\right)$, hence

$$
\begin{aligned}
\left\|\eta_{N}^{(6, B)}\right\|_{L^{2}}^{2} & \leq \sum_{(i, j) \in H_{1,2}^{(N)}}\left|\partial_{1} \partial_{2} g\left(\widetilde{s}_{i}^{(N)}, \widetilde{t}_{j}^{(N)}\right)-\partial_{1} \partial_{2} g\left(s_{i}^{(N)}, t_{j}^{(N)}\right)\right|^{2}\left(\Delta s_{i}^{(N)}\right)\left(\Delta t_{j}^{(N)}\right) \\
& \leq \lambda(G) \sup _{\substack{(s, t),(\widetilde{s}, \widetilde{t}) \in G \\
|s-\widetilde{s}|,\left|\gamma^{-1}(\widetilde{t})-\gamma^{-1}(t)\right| \leqslant\left|\mathcal{P}_{N}\right|}}\left|\partial_{1} \partial_{2} g(\widetilde{s}, \widetilde{t})-\partial_{1} \partial_{2} g(s, t)\right|^{2} \rightarrow \infty
\end{aligned}
$$

as $N \rightarrow \infty$

\section{Acknowledgements}

The authors are indebted to the unknown referee for the useful remarks and comments. 
Research has been supported by the Hungarian Scientific Research Fund under Grant No. OTKA-T079128/2009.

\section{References}

[1] K. Kuroda and H. Manaka, The interface of the Ising model and the Brownian sheet, J. Stat. Phys. 47 (1987), 979-984.

[2] J.F. Douglas, Swelling and growth of polymers, membranes, and sponges, Phys. Rev. E 54 (1996), 2677-2689.

[3] R. Goldstein, The term structure of interest rates as a random field, Rev. Financ. Stud. 13 (2000), 365-384.

[4] E. Biffis and P. Millossovich, A bidimensional approach to mortality risk, Decisions Econ. Finan. 29 (2006), 71-94.

[5] A.V. Carter, A continuous Gaussian approximation to a nonparametric regression in two dimensions, Bernoulli 12 (2006), 143-156.

6] H.H. Kuo, Gaussian Measures in Banach Spaces, Springer-Verlag, Berlin, 1975.

[7] I.A. Ibragimov and R.Z. Has'minskii, Statistical Estimation. Asymptotic Theory, SpringerVerlag, New York, 1981.

[8] E. Wong and M. Zakai, Likelihood ratios and transformations of probability associated with two-parameter Wiener processes, Z. Wahrsch. Verw. Gebiete 40 (1977), 283-308.

[9] Yu.A. Rozanov, Gaussian Infinite-dimensional Distributions, (in Russian), Tr. Mat. Inst. Akad. Nauk SSSR, Nauka, Moscow, 1968.

[10] Yu.A. Rozanov, Some boundary problems for generalized random fields, Theor. Probab. Appl. 35 (1990), 707-724.

[11] N.M. Arató, Mean estimation of Brownian sheet, Comput. Math. Appl. 33 (1997), 13-25.

[12] S. Baran, G. Pap and M.v. Zuijlen, Estimation of the mean of a Wiener sheet. Stat. Inference Stoch. Process. 7 (2004), 279-304.

[13] S. Baran, G. Pap and M.v. Zuijlen, Estimation of the mean of stationary and nonstationary Ornstein-Uhlenbeck processes and sheets, Comput. Math. Appl. 45 (2003), 563-579.

[14] M. Arató, Linear Stochastic Systems with Constant Coefficients. A Statistical Approach, Springer-Verlag, Berlin, 1982 (in Russian: Nauka, Moscow, 1989). 